\title{
Set-Valued Haezendonck-Goovaerts Risk Measure and Its Properties
}

\author{
Yu Feng, ${ }^{1}$ Yichuan Dong, ${ }^{1}$ and Jia-Bao Liu' ${ }^{2}$ \\ ${ }^{1}$ School of Mathematics and Statistics, Wuhan University, Wuhan, Hubei 430072, China \\ ${ }^{2}$ School of Mathematics and Physics, Anhui Jianzhu University, Hefei, Anhui 230601, China \\ Correspondence should be addressed to Jia-Bao Liu; liujiabaoad@163.com
}

Received 28 June 2017; Revised 20 August 2017; Accepted 13 September 2017; Published 15 October 2017

Academic Editor: Chris Goodrich

Copyright (c) 2017 Yu Feng et al. This is an open access article distributed under the Creative Commons Attribution License, which permits unrestricted use, distribution, and reproduction in any medium, provided the original work is properly cited.

\begin{abstract}
We propose a new set-valued risk measure, which is called set-valued Haezendonck-Goovaerts risk measure. First, we construct the set-valued Haezendonck-Goovaerts risk measure and then provide an equivalent representation. The properties of the set-valued Haezendonck-Goovaerts risk measure are investigated, which show that the set-valued Haezendonck-Goovaerts risk measure is coherent. Finally, an example of set-valued Haezendonck-Goovaerts risk measure is given, which exhibits the fact that the set-valued average value at risk is a particular case of the set-valued Haezendonck-Goovaerts risk measures.
\end{abstract}

\section{Introduction}

Premium principles are important and basic issues in insurance and actuarial science. Among various premium principles, the so-called Haezendonck-Goovaerts premium principle (also called Haezendonck-Goovaerts risk measure) has especially been attracting the attention of the insurance community and the financial community, not only because the Haezendonck-Goovaerts risk measure is a coherent risk measure introduced by Artzner et al. [1] but also because it has good properties and a wide range of applications in the finance field and the insurance field. Therefore, it plays an important role in both financial community and insurance community. Haezendonck and Goovaerts [2] introduced a new premium principle based on Orlicz norms. Goovaerts et al. [3] further studied the premium principle by Haezendonck and Goovaerts [2] and called it the Haezendonck risk measure in honor of the late Haezendonck. HaezendonckGoovaerts risk measure describes a class of concrete risk measures which derived by different parameters. Bellini and Rosazza Gianin [4] further studied Haezendonck risk measure. Bellini and Rosazza Gianin [5] continued the study of Haezendonck and used the terminology of HaezendonckGoovaerts risk measure in order to better acknowledge both authors' contribution in the seminal papers by Haezendonck and Goovaerts [2] and Goovaerts et al. [3]. All the above premium principles are for single risks (i.e., single claims). For more studies about insurance in recent years, see [6-11] and the references cited therein.

In some sense, risk measures can be considered as the counterpart of premium principles; see Bellini and Rosazza Gianin [4]. Meanwhile, in finance, in order to evaluate the risk of multivariate risks, with possible dependence between separate individual risks, the so-called scalar multivariate risk measures have been studied. For scalar multivariate coherent and convex risk measures, see Burgert and Rüschendorf [12], Rüschendorf [13], and the references cited therein. Moreover, besides the possible dependence between the separate individual risks, if the assets in different security markets are also involved in the multivariate risks, the so-called set-valued risk measures have been suggested and investigated. To be specific, the set-valued risk measure was firstly introduced to allow for random portfolios valued in $\mathbf{R}^{d}$ and relate each component of this portfolio to a specific security market. The motivation is that most of the investors are not able to aggregate their portfolio because of transaction costs between different security markets [14]. For the set-valued multivariate coherent and convex risk measures, see Jouini et al. [14], 
Hamel et al. [15], and Ararat et al. [16]. For more study about risk measure and set-valued risk measure in recent years, see $[3,17-20]$ and the references cited therein.

The existing Haezendonck-Goovaerts risk measure only takes into account a single risk position. When investors tend to consider a portfolio which contains different currencies or securities in different markets, the problems of conveniences and transaction costs can not be solved by the existing Haezendonck-Goovaerts risk measure. In the present paper, we propose the set-valued Haezendonck-Goovaerts risk measures for multivariate risks (i.e., risk portfolio vectors) to measure the risk of financial portfolios taking into account the transaction costs. Also, the set-valued HaezendonckGoovaerts risk measure provides a convenient and effective way to compare different portfolios and their margins. By construction, we will define the set-valued HaezendonckGoovaerts risk measure. Then an equivalent representation is provided, whose properties are investigated. It turns out that the set-valued Haezendonck-Goovaerts risk measures are coherent. Finally, an example is given, which shows that the set-valued average valued at risk by Hamel et al. [15] is a particular case of the set-valued Haezendonck-Goovaerts risk measures.

The rest of this paper is organized as follows. In Section 2, we briefly introduce the preliminaries. Section 3 is the study of the regular set-valued Haezendonck-Goovaerts risk measures. Section 4 falls on an extended form of setvalued Haezendonck-Goovaerts risk measures. Section 5 is an example of set-valued Haezendonck-Goovaerts risk measures, which also shows that the set-valued average value at risk is a particular case of the set-valued HaezendonckGoovaerts risk measures.

\section{Preliminaries}

Let $(\Omega, \mathscr{F}, P)$ be a probability space and $d \geq 1$ a positive integer. A multivariate random variable (understood as a financial portfolio) is an $\mathscr{F}$-measurable function $\mathbf{X}: \Omega \rightarrow \mathbf{R}^{d}$ for $d \geq 2$. In this paper, we regard $\mathbf{X}$ as "loss." When $d=1$, the random variate is called univariate. Denote by $L_{d}^{0}:=$ $L_{d}^{0}(\Omega, \mathscr{F}, P)$ the linear space of the equivalence classes (with respect to the probability $P$ ) of $\mathbf{R}^{d}$-valued random variables. An element $\mathbf{X} \in L_{d}^{0}$ has components $X_{1}, \ldots, X_{d}$ in $L^{0}:=$ $L_{1}^{0}$, where each component $X_{i}, 1 \leq i \leq d$, is regarded as a single financial position. Denote by $\left(L_{d}^{0}\right)_{+}$the set of $\mathbf{R}^{d}$ valued random variables with $P$-almost surely nonnegative components and by $L_{d}^{1}:=L_{d}^{1}(\Omega, \mathscr{F}, P)$ the linear space of all $\mathbf{X}=\left(X_{1}, \ldots, X_{d}\right) \in L_{d}^{0}$ with $\int_{\Omega} X_{i} d P<+\infty, 1 \leq i \leq d$. We also define $E[\mathbf{X}]=\left(E X_{1}, \ldots, E X_{d}\right)^{T}$ for $\mathbf{X} \in L_{d}^{1}$, the transpose of row vector $\left(E X_{1}, \ldots, E X_{d}\right)$. Define $\left(L_{d}^{1}\right)_{+}=L_{d}^{1} \cap$ $\left(L_{d}^{0}\right)_{+}$. If $d=1$, we write $L^{0}, L_{+}^{0}$, and $L_{+}^{1}$ for $L_{1}^{0},\left(L_{d}^{0}\right)_{+}$, and $\left(L_{d}^{1}\right)_{+}$, respectively. $x^{+}$stands for $\max (x, 0)$ for $x \in \mathbf{R}$. Write $\mathbf{R}^{+}:=[0,+\infty)$ and $\mathbf{M}:=\mathbf{R}^{m} \times\{0\}^{d-m}$.

A Young Function $\Phi$ is a mapping from $\mathbf{R}^{+}$to $\mathbf{R}^{+}$ that can be written as an integral of the form $\Phi(x)=$ $\int_{0}^{x} g(t) d t$, where $g$ is a left-continuous, monotone increasing real-valued function on $\mathbf{R}^{+}$with $g(0)=0$ and $\lim _{x \rightarrow \infty} g(x)=$ $\infty$. We say that $g$ is the kernel of the Young Function $\Phi$. It is easy to see that $\Phi$ is continuous, convex, and strictly increasing on the set $\{\Phi>0\}$. We say a Young Function is normalized if $\Phi(1)=1$. Any Young Function can be normalized by taking $\Phi(x) / \Phi(1)$.

The prototype of Haezendonck-Goovaerts risk measure is called the Orlicz premium principle studied by Haezendonck and Goovaerts [2]. The Orlicz premium principle is defined on Orlicz space.

Definition 1. Let $\Phi$ be a Young Function; the set $L_{\Phi}$ of random variables $\mathbf{X}$ defined as follows is called an Orlicz space:

$$
L_{\Phi}:=\left\{\mathbf{X} \mid E\left[\Phi\left(\frac{|\mathbf{X}|}{a}\right)\right] \leq 1, \text { for some } a>0\right\} .
$$

Let $\|\mathbf{X}\|_{\Phi}:=\inf \{a>0 \mid E[\Phi(|\mathbf{X}| / a)] \leq 1\} ;\|\mathbf{X}\|_{\Phi}$ is called the Orlicz norm of $\mathbf{X}$ corresponding to the Young Function $\Phi$.

For more information about Orlicz space as well as Orlicz heart, see Haezendonck and Goovaerts [2] and Bellini and Rosazza Gianin [5].

The following definition of Orlicz premium principle is from Haezendonck and Goovaerts [2].

Definition 2. Let $\mathbf{X} \in L_{\infty}^{+}$with $\mathbf{X}$ not being equal to zero a.s. and $H(\mathbf{X})$ be the solution of the equation $\Psi(x)=$ $E[\Phi(\mathbf{X} / x)]=1$. By convention $H(0) \triangleq 0, H(\cdot)$ is called the Orlicz premium principle on $L_{\infty}^{+}$.

Note that the solution $H(\mathbf{X})$ exists uniquely and that it is exactly the Orlicz norm $\|\mathbf{X}\|_{\Phi}$ as in Definition 1. Moreover, $H(\cdot)$ is a coherent risk measure by Artzner et al. [1]. For more details, see Haezendonck and Goovaerts [2].

Given $\alpha \in(0,1)$, Bellini and Rossaza Gianin [4] introduced the following Haezendonck-Goovaerts risk measure.

Definition 3. Let $\alpha \in(0,1)$, for $\mathbf{X} \in L^{\infty}, x \in \mathbf{R}$ with $x<$ $\operatorname{esssup}(\mathbf{X}) ; \pi_{\alpha}(\mathbf{X}, x)$ is denoted as the solution of the equation

$$
E\left[\Phi\left(\frac{(\mathbf{X}-x)^{+}}{\pi_{\alpha}(\mathbf{X}, x)-x}\right)\right]=1-\alpha
$$

$\pi_{\alpha}(\mathbf{X}, x)$ is called Haezendonck-Goovaerts risk measure with respect to $\mathbf{X} \in L^{\infty}$.

Note that $\pi_{\alpha}(\mathbf{X}, x)=H\left((\mathbf{X}-x)^{+}\right)+x$. For more studies, see Bellini and Rossaza Gianin $[4,5]$.

Next, we introduce the set-valued risk measures. The power set of $\mathbf{R}^{n}$ is denoted by $\mathscr{P}\left(\mathbf{R}^{n}\right)$. In general, a $(d-n)$ set-valued risk measure is any mapping from $L_{d}^{\infty}$ into $\mathscr{P}\left(\mathbf{R}^{n}\right)$, for $n \leq d$.

Definition 4. A $(d-n)$ set-valued risk measure $R: L_{d}^{\infty} \rightarrow$ $\mathscr{P}\left(\mathbf{R}^{n}\right)$ is coherent; it satisfies the following axioms:

(A0) For all $\mathbf{X} \in L_{d}^{\infty}, R(\mathbf{X})$ is closed and $0 \in R(0) \neq \mathbf{R}^{n}$.

(A1) For all $\mathbf{X} \in L_{d}^{\infty}, \mathbf{X} \geq 0 P-$ a.s. $\Rightarrow R(0) \subset R(\mathbf{X})$.

(A2) For all $\mathbf{X}, \mathbf{Y} \in L_{d}^{\infty}, R(\mathbf{X})+R(\mathbf{Y}) \subset R(\mathbf{X}+\mathbf{Y})$. 
(A3) For all $t>0$ and $\mathbf{X} \in L_{d}^{\infty}, R(t \mathbf{X})=t R(\mathbf{X})$.

(A4) For all $x \in \mathbf{R}^{n}$ and $\mathbf{X} \in L_{d}^{\infty}, R(\mathbf{X}+x)=\{-x\}+R(\mathbf{X})$.

For more details about the definition above, see Jouini et al. [14].

Hamel et al. [15] introduced the so-called set-valued average value at risk, which is coherent.

Definition 5. Let $\alpha \in(0,1]^{d}$ and $\mathbf{X} \in L_{d}^{\infty}$; the average value at risk of $\mathbf{X}$ is defined as

$$
\begin{aligned}
& \mathrm{AV} @ R_{\alpha}(\mathbf{X}):=\left\{\operatorname{diag}(\alpha)^{-1} E[\mathbf{Z}]-z \mid \mathbf{Z} \in\left(L_{d}^{1}\right)_{+}, \mathbf{X}\right. \\
& \left.\quad+\mathbf{Z}-z \in\left(L_{d}^{0}\right)_{+}, z \in \mathbf{R}^{d}\right\} .
\end{aligned}
$$

It has an equivalent representation as follows:

$$
\begin{aligned}
\operatorname{AV} @ R_{\alpha}(\mathbf{X})= & {\left[\inf _{z_{i} \in \mathbf{R}}\left(\frac{1}{\alpha_{i}} E\left[\left(-X_{i}+z_{i}\right)^{+}\right]-z_{i}\right)\right]_{i=1}^{d} } \\
& +\mathbf{R}^{m} \times\{0\}^{d-m} .
\end{aligned}
$$

\section{The Regular Set-Valued Haezendonck-Goovaerts Risk Measure}

In this section, we will give the definition of set-valued Haezendonck-Goovaerts risk measure. Meanwhile, its properties and an equivalent representation are provided.

Definition 6. Let $\alpha \in(0,1)^{d}$. For $\mathbf{X} \in L_{d}^{0}$, let $H_{\alpha}(\mathbf{X})$ be the solution of equation $E\left[\Phi\left(\mathbf{X} / H_{\alpha}(\mathbf{X})\right)\right]=1-\alpha$. The set-valued Haezendonck-Goovaerts risk measure is defined as follows:

$$
\begin{aligned}
& \mathrm{HG}_{\alpha}(\mathbf{X}):=\left\{H_{\alpha}(\mathbf{Z})+z \mid \mathbf{Z} \in\left(L_{d}^{1}\right)_{+},-\mathbf{X}+\mathbf{Z}+z\right. \\
& \left.\in\left(L_{d}^{0}\right)_{+}, z \in \mathbf{R}^{d}\right\} \cap \mathbf{M} .
\end{aligned}
$$

Here $H_{\alpha}(\mathbf{Z})+z:=\left(H_{\alpha_{i}}\left(Z_{i}\right)+z_{i}\right)_{i=1}^{d}$.

Remark 7. The intersection with $\mathbf{M}$ in Definition 1 has an interpretation as follows. When measuring the risk of portfolio $\mathbf{X}$, we should work out the set of all the margins that can cancel the risk of portfolio $\mathbf{X}$. After intersecting the set $\mathbf{M}$, $\mathrm{HG}_{\alpha}$ not only shows the valid margins but also aggregates the margins. It aggregates the valid margins from $d$-dimension to $m$-dimension valid margins and the other $(d-m)$-dimension should be zero. Aggregating margins has plenty of financial explanations. For example, different elements of the vector represent the amount in different currencies. Suppose that there are $m$ kinds of currencies in the vector margin. It is unnecessary for the regulator to ask investor for $d$-dimension vector margin. They could aggregate some elements, which are in the same currency, into one new element of the vector margin. This idea also makes sense when considering the margins needed by different departments in a company. The leader of this company may just deliver the sum of margins of different departments. For more details, see Jouini et al. [14].
Proposition 8. The set-valued Haezendonck-Goovaerts risk measure defined in Definition 6 is of the following form:

$$
\begin{aligned}
H G_{\alpha}(\mathbf{X})= & {\left[\inf _{z_{i} \in \mathbf{R}}\left(z_{i}+H_{\alpha_{i}}\left(\left(X_{i}-z_{i}\right)^{+}\right)\right)\right]_{i=1}^{d}+\mathbf{R}_{+}^{m} } \\
& \times\{0\}^{d-m} .
\end{aligned}
$$

One denotes $H G_{i}\left(X_{i}\right)=\inf _{z_{i} \in \mathbf{R}}\left(z_{i}+H_{\alpha_{i}}\left(\left(X_{i}-z_{i}\right)^{+}\right)\right)+\mathbf{R}_{+}$.

Proof. The condition of Definition 6 equals $\mathbf{Z} \geq(\mathbf{X}-z)^{+}, z \in$ $\mathbf{R}^{d}$. Then we have

$$
\begin{aligned}
& \mathrm{HG}_{\alpha}(\mathbf{X})=\left\{H_{\alpha}(\mathbf{Z})+z \mid \mathbf{Z} \in\left(L_{d}^{1}\right)_{+},-\mathbf{X}+\mathbf{Z}+z\right. \\
& \left.\in\left(L_{d}^{0}\right)_{+}, z \in \mathbf{R}^{d}\right\} \cap \mathbf{M}=\left\{H_{\alpha}(\mathbf{Z})+z \mid \mathbf{Z}\right. \\
& \left.\geq(\mathbf{X}-\mathbf{Z})^{+}, z \in \mathbf{R}^{d}\right\} .
\end{aligned}
$$

According to the monotonicity of $H_{\alpha}(\cdot)$, we have

$$
\begin{aligned}
& \mathrm{HG}_{\alpha}(\mathbf{X})=\left\{H_{\alpha}(\mathbf{Z})+z \mid H_{\alpha}(\mathbf{Z}) \geq H_{\alpha}\left((\mathbf{X}-z)^{+}\right), z\right. \\
& \left.\quad \in \mathbf{R}^{d}\right\} \cap \mathbf{M}=\left\{H_{\alpha}(\mathbf{Z})+z \mid H_{\alpha}(\mathbf{Z})+z\right. \\
& \left.\quad \geq H_{\alpha}\left((\mathbf{X}-z)^{+}\right)+z, z \in \mathbf{R}^{d}\right\} \cap \mathbf{M} .
\end{aligned}
$$

Hence, we obtain

$$
\begin{aligned}
\mathrm{HG}_{\alpha}(\mathbf{X})= & {\left[\inf _{z_{i} \in \mathbf{R}}\left(H_{\alpha_{i}}\left(\left(X_{i}-z_{i}\right)^{+}\right)+z_{i}\right)\right]_{i=1}^{d}+\mathbf{R}_{+}^{m} } \\
& \times\{0\}^{d-m} .
\end{aligned}
$$

Proposition 9. The function $\mathbf{X} \mapsto H G_{\alpha}(\mathbf{X})$ satisfies the following properties:

(a) It is positive homogeneous; that is, for any $\mathbf{X} \in L_{d}^{0}$ and any $s>0$, we have $H G_{\alpha}(s \mathbf{X})=s H G_{\alpha}(\mathbf{X})$.

(b) It is subadditive; that is, for any $\mathbf{X}^{1}, \mathbf{X}^{2} \in L_{d}^{0}$, we have $H G_{\alpha}\left(\mathbf{X}^{1}\right)+H G_{\alpha}\left(\mathbf{X}^{2}\right) \subseteq H G_{\alpha}\left(\mathbf{X}^{1}+\mathbf{X}^{2}\right)$.

(c) It is $\mathbf{M}$-translative; that is, for any $\mathbf{X} \in L_{d}^{0}$ and any $u \in \mathbf{M}$, we have $H G_{\alpha}(\mathbf{X}+\bar{u})=H G_{\alpha}(\mathbf{X})+u$; here $\bar{u}=u \times\{0\}^{d-m}$.

(d) It is monotone with respect to $\left(L_{d}^{0}\right)_{+}$; that is, for any $\mathbf{X}^{1}, \mathbf{X}^{2} \in\left(L_{d}^{0}\right)_{+}$and $\mathbf{X}^{2} \geq \mathbf{X}^{1}$ which means $\mathbf{X}^{2}-\mathbf{X}^{1} \epsilon$ $\left(L_{d}^{0}\right)_{+}$, we have $H G_{\alpha}\left(\mathbf{X}^{2}\right) \subseteq H G_{\alpha}\left(\mathbf{X}^{1}\right)$.

(e) It is convex and satisfies $H G_{\alpha}(\mathbf{X})+\mathbf{M}_{+}=H G_{\alpha}(\mathbf{X})$. Particularly, $\mathrm{HG}_{\alpha}(\mathbf{0})$ is a convex cone. 
Remark 10. Properties (a)-(d) ensure that set-valued Haezendonck-Goovaerts risk measure defined in Definition 6 is a coherent set-valued risk measure.

Proof of Proposition 9. (a)

$$
\begin{aligned}
& s \mathrm{HG}_{\alpha}(\mathbf{X})=s\left\{H_{\alpha}(\mathbf{Z})+z \mid \mathbf{Z} \in\left(L_{d}^{1}\right)_{+},-\mathbf{X}+\mathbf{Z}+z\right. \\
& \left.\in\left(L_{d}^{0}\right)_{+}, z \in \mathbf{R}^{d}\right\} \cap \mathbf{M}=\left\{H_{\alpha}(s \mathbf{Z})+s z \mid \mathbf{Z}\right. \\
& \left.\in\left(L_{d}^{1}\right)_{+},-\mathbf{X}+\mathbf{Z}+z \in\left(L_{d}^{0}\right)_{+}, z \in \mathbf{R}^{d}\right\} \cap \mathbf{M} \\
& \quad=\left\{H_{\alpha}(\mathbf{Z})+z \mid \frac{\mathbf{Z}}{s} \in\left(L_{d}^{1}\right)_{+},-\mathbf{X}+\frac{\mathbf{Z}}{s}+\frac{z}{s}\right. \\
& \left.\in\left(L_{d}^{0}\right)_{+}, \frac{z}{s} \in \mathbf{R}^{d}\right\} \cap \mathbf{M}=\left\{H_{\alpha}(\mathbf{Z})+z \mid \frac{\mathbf{Z}}{s}\right. \\
& \left.\quad \in\left(L_{d}^{1}\right)_{+}, \frac{1}{s}(-s \mathbf{X}+\mathbf{Z}+z) \in\left(L_{d}^{0}\right)_{+}, \frac{z}{s} \in \mathbf{R}^{d}\right\} \\
& \cap \mathbf{M}^{\prime}=\left\{H_{\alpha}(\mathbf{Z})+z \mid \mathbf{Z} \in\left(L_{d}^{1}\right)_{+},-s \mathbf{X}+\mathbf{Z}+z\right. \\
& \left.\in\left(L_{d}^{0}\right)_{+}, z \in \mathbf{R}^{d}\right\} \cap \mathbf{M}=\mathrm{HG}_{\alpha}(s \mathbf{X}) .
\end{aligned}
$$

(b)

$$
\begin{aligned}
& \mathrm{HG}_{\alpha}\left(\mathbf{X}^{1}\right)+\mathrm{HG}_{\alpha}\left(\mathbf{X}^{2}\right)=\left\{H_{\alpha}\left(\mathbf{Z}^{1}\right)+z^{1}+H_{\alpha}\left(\mathbf{Z}^{2}\right)\right. \\
& +z^{2} \mid \mathbf{Z}^{1} \in\left(L_{d}^{1}\right)_{+}, \mathbf{Z}^{2} \in\left(L_{d}^{1}\right)_{+},-\mathbf{X}^{1}+\mathbf{Z}^{1}+z^{1} \\
& \quad \in\left(L_{d}^{0}\right)_{+},-\mathbf{X}^{2}+\mathbf{Z}^{2}+z^{2} \in\left(L_{d}^{0}\right)_{+}, z^{1} \in \mathbf{R}^{d}, z^{2} \\
& \left.\quad \in \mathbf{R}^{d}\right\} \cap \mathbf{M} \subseteq\left\{H_{\alpha}\left(\mathbf{Z}^{1}\right)+z^{1}+H_{\alpha}\left(\mathbf{Z}^{2}\right)+z^{2} \mid \mathbf{Z}^{1}\right. \\
& +\mathbf{Z}^{2} \in\left(L_{d}^{1}\right)_{+},-\mathbf{X}^{1}+\mathbf{Z}^{1}+z^{1}-\mathbf{X}^{2}+\mathbf{Z}^{2}+z^{2} \\
& \left.\quad\left(L_{d}^{0}\right)_{+}, z^{1}+z^{2} \in \mathbf{R}^{d}\right\} \cap \mathbf{M} \subseteq\left\{H_{\alpha}\left(\mathbf{Z}^{1}+\mathbf{Z}^{2}\right)\right. \\
& +z^{1}+z^{2} \mid \mathbf{Z}^{1}+\mathbf{Z}^{2} \in\left(L_{d}^{1}\right)_{+},-\mathbf{X}^{1}+\mathbf{Z}^{1}+z^{1}-\mathbf{X}^{2} \\
& \left.+\mathbf{Z}^{2}+z^{2} \in\left(L_{d}^{0}\right)_{+}, z^{1}+z^{2} \in \mathbf{R}^{d}\right\} \cap \mathbf{M} \\
& =\mathrm{HG}_{\alpha}\left(\mathbf{X}^{1}+\mathbf{X}^{2}\right),
\end{aligned}
$$

where the second inclusion holds because of the subadditivity and the positive homogeneity of $H_{\alpha}(\cdot)$ (see Bellini and Rossaza Gianin [4]); we have

$$
\begin{array}{r}
H_{\alpha}\left(\mathbf{Z}^{1}\right)+H_{\alpha}\left(\mathbf{Z}^{2}\right)+z^{1}+z^{2} \\
\geq H_{\alpha}\left(\mathbf{Z}^{1}+\mathbf{Z}^{2}\right)+z^{1}+z^{2}
\end{array}
$$

which implies

$$
\begin{aligned}
& H_{\alpha}\left(\mathbf{Z}^{1}\right)+H_{\alpha}\left(\mathbf{Z}^{2}\right)+z^{1}+z^{2} \\
& \quad=a\left[H_{\alpha}\left(\mathbf{Z}^{1}+\mathbf{Z}^{2}\right)\right]+z^{1}+z^{2} \\
& \quad=H_{\alpha}\left(a\left(\mathbf{Z}^{1}+\mathbf{Z}^{2}\right)\right)+z^{1}+z^{2} .
\end{aligned}
$$

(c) For any $\mathbf{U} \in\left(L_{d}^{0}\right)_{+}$, we have $\mathbf{U}+\left(L_{d}^{0}\right)_{+} \subseteq\left(L_{d}^{0}\right)_{+}$.

$$
\begin{aligned}
& \mathrm{HG}_{\alpha}(\mathbf{X}+\mathbf{U})=\left\{H_{\alpha}(\mathbf{Z})+z \mid \mathbf{Z} \in\left(L_{d}^{1}\right)_{+},-\mathbf{X}+\mathbf{Z}\right. \\
& \left.+z-\mathbf{U} \in\left(L_{d}^{0}\right)_{+}, z \in \mathbf{R}^{d}\right\} \cap \mathbf{M}=\left\{H_{\alpha}(\mathbf{Z})+z \mid \mathbf{Z}\right. \\
& \left.\quad \in\left(L_{d}^{1}\right)_{+},-\mathbf{X}+\mathbf{Z}+z \in \mathbf{U}+\left(L_{d}^{0}\right)_{+}, z \in \mathbf{R}^{d}\right\} \cap \mathbf{M} \\
& \quad \subseteq\left\{H_{\alpha}(\mathbf{Z})+z \mid \mathbf{Z} \in\left(L_{d}^{1}\right)_{+},-\mathbf{X}+\mathbf{Z}+z\right. \\
& \left.\quad \in\left(L_{d}^{0}\right)_{+}, z \in \mathbf{R}^{d}\right\} \cap \mathbf{M}=\mathrm{HG}_{\alpha}(\mathbf{X}) .
\end{aligned}
$$

So for any $\mathbf{X}^{2} \geq \mathbf{X}^{1}$, we have $\mathrm{HG}_{\alpha}\left(\mathbf{X}^{2}\right) \subset \mathrm{HG}_{\alpha}\left(\mathbf{X}^{1}\right)$.

(d)

$$
\begin{aligned}
& \mathrm{HG}_{\alpha}(\mathbf{X}+\bar{u})=\left\{H_{\alpha}(\mathbf{Z})+z \mid \mathbf{Z} \in\left(L_{d}^{1}\right)_{+},-\mathbf{X}-\bar{u}\right. \\
& \left.+\mathbf{Z}+z \in\left(L_{d}^{0}\right)_{+}, z \in \mathbf{R}^{d}\right\} \cap \mathbf{M}=\left\{H_{\alpha}(\mathbf{Z})+z \mid \mathbf{Z}\right. \\
& \in\left(L_{d}^{1}\right)_{+},-\mathbf{X}+\mathbf{Z}+(z-\bar{u}) \in \mathbf{U}+\left(L_{d}^{0}\right)_{+}, z-\bar{u} \\
& \left.\in \mathbf{R}^{d}\right\} \cap \mathbf{M}=\left\{H_{\alpha}(\mathbf{Z})+(z-\bar{u})+\bar{u} \mid \mathbf{Z} \in\left(L_{d}^{1}\right)_{+},\right. \\
& \left.-\mathbf{X}_{+} \mathbf{Z}+(z-\bar{u}) \in\left(L_{d}^{0}\right)_{+}, z-\bar{u} \in \mathbf{R}^{d}\right\} \cap \mathbf{M} \\
& =\mathrm{HG}_{\alpha}(\mathbf{X})+u .
\end{aligned}
$$

(e) It is obvious.

The proof of Proposition 9 is complete.

Before ending this section, we will introduce the concept of scalarization of set-valued Haezendonck-Goovaerts risk measure. The relationship between (5) and (6) will also be further illustrated by scalarization of set-valued HaezendonckGoovaerts risk measure. For simplicity, we denote the equivalent representation of set-valued Haezendonck-Goovaerts risk measure in (6) by $\mathrm{HG}_{\alpha}^{\mathrm{sca}}(\mathbf{X})$.

In the following, we assume that $\mathrm{HG}_{\alpha}^{\text {sca }}(\mathbf{X})$ is a nonempty closed set. Besides, it is also convex. So it is the intersection of all closed half-space including it. Such a half-space has an element $w \in\left(\mathbf{R}_{+}^{d} \cap \mathbf{M}\right)^{+} \backslash\{0\}=\left(\mathbf{R}_{+}^{d}+\mathbf{M}^{\perp}\right) \backslash\{0\}$. Then we have the following relationship:

$$
u \in \mathrm{HG}_{\alpha}(\mathbf{X}) \Longleftrightarrow \forall w \in\left(\mathbf{R}_{+}^{d}+\mathbf{M}^{\perp}\right) \backslash\{0\}: w^{T} u \geq \inf \left\{w^{T} x \mid x \in \mathrm{HG}_{\alpha}(\mathbf{X})\right\}
$$


Now we consider a real-valued function extended by $\mathrm{HG}_{\alpha}(\mathbf{X})$ :

$$
\mathbf{X} \longmapsto \phi_{\mathrm{HG}_{\alpha}(\mathbf{X}), w}(\mathbf{X})=\inf \left\{w^{T} u \mid u \in \mathrm{HG}_{\alpha}(\mathbf{X})\right\}
$$

Let any $v \in \mathbf{M}^{\perp}$; we can get that $\phi_{\mathrm{HG}_{\alpha}(\mathbf{X}), w+v}=\phi_{\mathrm{HG}_{\alpha}(\mathbf{X}), w}$ since $\mathrm{HG}_{\alpha}(\mathbf{X}) \subseteq \mathbf{M}$ for all $\mathbf{X} \in L_{d}^{0}$. Then we can restrict the function $\phi_{\mathrm{HG}_{\alpha}(\mathbf{X}), w}$ to those with $w \in \mathbf{R}_{+}^{d}$ and obtain the following relationship:

$$
u \in \mathrm{HG}_{\alpha}(\mathbf{X}) \Longleftrightarrow \forall w \in \mathbf{R}_{+}^{d}, \forall v \in \mathbf{M}^{\perp}, w+v \neq 0:(w+v)^{T} u \geq \phi_{\mathrm{HG}_{\alpha}(\mathbf{X}), w}(\mathbf{X}) .
$$

Furthermore, it is not difficult to restrict the functions $\phi_{\mathrm{HG}_{\alpha}(\mathbf{X}), w}$ to those with $w \in B(1)$, where $B(1)$ is defined as $B(1):=\left\{w \in \mathbf{R}_{+}^{d} \mid \sum_{i=1}^{d} w_{i}=1\right\}$, and we obtain

$$
u \in \mathrm{HG}_{\alpha}(\mathbf{X}) \Longleftrightarrow \forall w \in B(1), \forall v \in \mathbf{M}^{\perp}, w+v \neq 0:(w+v)^{T} u \geq \phi_{\mathrm{HG}_{\alpha}(\mathbf{X}), w}(\mathbf{X})
$$

Finally, it is easy to prove the next proposition which will show that the functions $\phi_{\mathrm{HG}_{\alpha}(\mathbf{X}), w}$ are composed of the scalar type $\mathrm{HG}_{\alpha}(\mathbf{X})$ (i.e., $\mathrm{HG}_{\alpha}^{\text {sca }}(\mathbf{X})$ ) functions for the components of $\mathbf{X}$.

Proposition 11. Let $H G_{\alpha}(\mathbf{X}) \neq \emptyset, w \in \mathbf{R}_{+}^{d} \backslash\{0\}$. Then

$$
\phi_{H G_{\alpha}(\mathbf{X}), w}(\mathbf{X})=\sum_{i=1}^{d} w_{i} H G_{i}\left(X_{i}\right)
$$

Remark 12. Proposition 11 together with the previous discussion, by the scalarization, provides the following representation of the set-valued Haezendonck-Goovaerts risk measure. If $\mathrm{HG}_{\alpha}(\mathbf{X})$ is closed, then

$$
\begin{aligned}
& \mathrm{HG}_{\alpha}(\mathbf{X})=\bigcap_{w \in B(1), v \in \mathbf{M}^{\perp}, w=v \neq 0}\left\{u \in \mathbf{R}^{d} \mid(w+v)^{T} u\right. \\
& \left.\geq \phi_{\mathrm{HG}_{\alpha}(\mathbf{X}), w}(\mathbf{X})\right\} .
\end{aligned}
$$

Remark 13. Proposition 11 means that the scalarization functions $\phi_{\mathrm{HG}_{\alpha}(\mathbf{X}), w}$ are convex combinations of the scalar Haezendonck-Goovaerts risk measure applied to the components of the portfolio $\mathbf{X}$. Moreover, $u \in \mathrm{HG}_{\alpha}(\mathbf{X})$ if and only if the convex combinations $w^{T} u$ cancel the risk of the convex combinations $w^{T} \mathbf{X}$ for all $w \in B(1)$. That explains why the multidimensional confidential level $\alpha$ may have different components.

The regulators and investors may have different attitudes towards the risk of different components of the portfolio $\mathbf{X}$. On the other hand, $w \in B(1)$ can be understood as weighting coefficients of the $d$ components of assets. When investors evaluate the risk of portfolio, they may have more and less favorable assets. Proposition 11 shows that the regulator does not care about the weighting which investors have chosen. The regulator only needs to be the safe side no matter the weighting that the investors choose.

\section{The Extended Set-Valued Haezendonck-Goovaerts Risk Measure}

In this section, we will give an extended set-valued Haezendonck-Goovaerts risk measure, which can match more complex market situation.

Definition 14. Let $\alpha \in(0,1)^{d}$, and let $K$ and $\widetilde{K}$ be closed convex cones which contain $\left(L_{d}^{0}\right)_{+}$and $\left(L_{d}^{1}\right)_{+}$, respectively. For $\mathbf{X} \in L_{d}^{0}$, the extended set-valued Haezendonck-Goovaerts risk measure is defined as follows.

$$
\begin{aligned}
& \mathrm{HG}_{\alpha}^{\text {ext }}(\mathbf{X}) \\
& \quad:=\left\{H_{\alpha}(\mathbf{Z})+z \mid \mathbf{Z} \in \widetilde{K},-\mathbf{X}+\mathbf{Z}+z \in K, z \in \mathbf{R}^{d}\right\}
\end{aligned}
$$

$\cap \mathbf{M}$.

Here, $H_{\alpha}(\mathbf{X})$ is the solution of $E\left[\Phi\left(\mathbf{X} / H_{\alpha}(\mathbf{X})\right)\right]=1-\alpha$.

Remark 15. Different closed convex cones represent different attitudes towards risks. $\left(L_{d}^{0}\right)_{+}$is a specific closed convex cone in $\left(L_{d}^{0}\right)$. When choosing $K=\left(L_{d}^{0}\right)_{+}$, the regulators (investors) consider $-\mathbf{X} \succeq_{K} 0$ (equivalently $-\mathbf{X} \geq 0$ ) as that $-\mathbf{X}$ is component-wise greater than 0 . They demand that each position of the portfolio $-\mathbf{X}$ can not be lost. When choosing a general closed convex cone $K,-\mathbf{X} \geq_{K} 0$ means that $-\mathbf{X} \in K$. In this case, the regulators (or investors) are risk appetite. They allow several positions to be negative and this portfolio is also acceptable to them.

Remark 16. There are pros and cons of the set-valued Haezendonck-Goovaerts risk measure compared with current risk measures like value at risk, scalar HaezendonckGoovaerts risk measure, and other coherent risk measures. The pros are that it provides a more convenient and effective way to compare different portfolios. For example, there are two-dimensional portfolios like $(5,6)$ and $(6,5)$. Two elements of these portfolios represent Dollar and Euro, 
respectively. It is not convenient to figure out which portfolio is greater than the other one within the framework of current scalar risk measure. But within the framework of set-valued Haezendonck-Goovaerts risk measure, one can immediately figure out which portfolio is greater by fixing a closed convex cone $K .(5,6)$ is greater than $(6,5)$ if and only if $(5-6,6-$ $5)=(-1,1) \in K$. That defines a partial order relationship (denoted by $\succeq_{K}$ ) between different portfolios, which is equal to normal partial order relationship $(\geq)$ by letting $K$ be equal to $\mathbf{R}_{+}^{d}$. Another superiority of set-valued HaezendonckGoovaerts risk measure is that it meets various investors' (or regulators') requirements and can be applied to a wide range of scenarios such as different levels of preference of risk and different financial markets. As we know, when using scalar Haezendonck-Goovaerts risk measure, regulators choose different risk preference by choosing different confidence level $\alpha$. Within set-valued Haezendonck-Goovaerts risk measure, investors can also choose a level of preference of risk by selecting the closed convex cone $K$. Supposing that there are two closed convex cones $K_{1}$ and $K_{2}$ with $K_{1} \subseteq K_{2}, K_{1}, K_{2} \supseteq \mathbf{R}_{+}^{d}$, $K_{1}$ relates to a stricter preference towards risks. Investors who select $K_{1}$ are conservative. The cons of set-valued Haezendonck-Goovaerts risk measure reflect that it has many parameters. So it is not easy to be used for general investors. Besides, its complex expression makes it difficult to compute.

By checking the proof of Proposition 8, we can steadily prove the following Proposition 17, which shows that the extended set-valued Haezendonck-Goovaerts risk measure has an equivalent representation.

Proposition 17. The extended set-valued HaezendonckGoovaerts risk measure has the following equivalent representation:

$$
\begin{aligned}
H G_{\alpha}^{\text {ext }}(\mathbf{X})= & {\left[\inf _{z_{i} \in \mathbf{R}}\left(z_{i}+H_{\alpha_{i}}\left(\left(X_{i}-z_{i}\right)^{+}\right)\right)\right]_{i=1}^{d}+\mathbf{C} } \\
& \times\{0\}^{d-m}
\end{aligned}
$$

where $\mathbf{C}$ is a closed convex cone which contains $\mathbf{R}_{+}^{m}$.

Remark 18. K, $\widetilde{K}$, and $\mathrm{C}$ are in the same level of preference of risk.

Proposition 19. The function $\mathbf{X} \mapsto H G_{\alpha}^{\text {ext }}(\mathbf{X})$ satisfies the following properties:

(a) It is positive homogeneous; that is, for any $\mathbf{X} \in L_{d}^{0}$ and any $s>0$, we have $H G_{\alpha}^{\text {ext }}(s \mathbf{X})=s H G^{\text {ext }}(\mathbf{X})$.

(b) It is subadditive; that is, for any $\mathbf{X}^{1}, \mathbf{X}^{2} \in L_{d}^{0}$, we have $H G_{\alpha}^{e x t}\left(\mathbf{X}^{1}\right)+H G_{\alpha}^{e x t}\left(\mathbf{X}^{2}\right) \subseteq H G_{\alpha}^{e x t}\left(\mathbf{X}^{1}+\mathbf{X}^{2}\right)$.

(c) It is $\mathbf{M}$-translative; that is, for any $\mathbf{X} \in L_{d}^{0}$ and any $u \in \mathbf{M}$, we have $H G_{\alpha}^{\text {ext }}(\mathbf{X}+\bar{u})=H G_{\alpha}^{\text {ext }}(\mathbf{X})+u$, here $\bar{u}=u \times\{0\}^{d-m}$.

(d) It is monotone with respect to $K$; that is, for any $\mathbf{X}^{1}, \mathbf{X}^{2} \in L_{d}^{0}$ and $\mathbf{X}^{2} \succeq_{K} \mathbf{X}^{1}$ which means $\mathbf{X}^{2}-\mathbf{X}^{1} \in K$, we have $H G_{\alpha}^{e x t}\left(\mathbf{X}^{2}\right) \subseteq H G_{\alpha}^{e x t}\left(\mathbf{X}^{1}\right)$. (e) It is convex and satisfies $H G_{\alpha}^{\text {ext }}(\mathbf{X})+\mathbf{M}_{+}=H G_{\alpha}^{\text {ext }}(\mathbf{X})$. Particularly, $\mathrm{HG}_{\alpha}^{\text {ext }}(\mathbf{0})$ is a convex cone.

Properties (a)-(d) ensure that $\mathrm{HG}_{\alpha}^{\text {ext }}(\cdot)$ is a set-valued coherent risk measure. The proof of Proposition 19 is similar to that of Proposition 9, and so it is omitted here.

\section{Computation and Example of Set-Valued Haezendonck-Goovaerts Risk Measure}

In this section, we will introduce the computation of setvalued Haezendonck-Goovaerts risk measure after the portfolio is given and the parameters are fixed. Let the portfolio contain three positions, that is, $d=3$. We suppose that there are three scenarios, that is, $N=3$ and $p=(1 / 2,1 / 3,1 / 6)$. The payoff is given by

$$
\begin{aligned}
& \mathbf{X}\left(\omega_{1}\right)=(-7,-4,-2)^{T}, \\
& \mathbf{X}\left(\omega_{2}\right)=(-5,3,2)^{T}, \\
& \mathbf{X}\left(\omega_{3}\right)=(4,-8,9)^{T} .
\end{aligned}
$$

We use the confidential level $\alpha=\left(\alpha_{1}, \alpha_{2}, \alpha_{3}\right)=(0.05,0.05$, $0.05)$. Furthermore, let $\mathbf{M}=\mathbf{R}^{3}$ and the Young Function $\Phi(x)=x$. Then a straightforward calculation shows that

$$
\mathrm{HG}_{\alpha}(\mathbf{X})=(4,3,9)^{T}+\mathbf{R}_{+}^{3} \text {. }
$$

Thus, the minimal risk compensating portfolio for a regulator (or investor) covers the worst case for the three assets which are 4 units, 3 units, and 9 units, respectively. Besides, any margin portfolio which belongs to $(4,3,9)^{T}+\mathbf{R}_{+}^{3}$ will be accepted by regulators (or investors).

As we know, in terms of scalar Haezendonck-Goovaerts risk measure, a particular case of Haezendonck-Goovaerts risk measure is the average value at risk. When Young Function $\Phi(x)=x$, Haezendonck-Goovaerts risk measure comes to the average value at risk. In the same way, set-valued Haezendonck-Goovaerts risk measure defines a class of risk measures. Different parameter leads to different set-valued risk measure. Among those set-valued risk measures, setvalued average value at risk is a typical example of set-valued Haezendonck-Goovaerts risk measure. Before ending this article, we will show this relationship between set-valued Haezendonck-Goovaerts risk measure and set-valued average valued at risk.

Let us start with the equation

$$
E\left[\Phi\left(\frac{\mathbf{X}}{H_{\alpha}(\mathbf{X})}\right)\right]=\alpha
$$


Let the Young Function $\Phi(x)=x$; this equation equals

$$
E\left[\frac{\mathbf{X}}{H_{\alpha}(\mathbf{X})}\right]=\alpha
$$

which leads to the solution

$$
H_{\alpha}(\mathbf{X})=\frac{E[\mathbf{X}]}{\alpha} .
$$

After taking into account this solution, the definition of setvalued Haezendonck-Goovaerts risk measure turns out to be

$$
\begin{aligned}
\mathrm{HG}_{\alpha}(\mathbf{X})= & {\left[\inf _{z_{i} \in \mathbf{R}}\left(\frac{1}{\alpha_{i}} E\left[\left(X_{i}-z_{i}\right)^{+}\right]+z_{i}\right)\right]_{i=1}^{d}+\mathbf{R}^{m} } \\
& \times\{0\}^{d-m},
\end{aligned}
$$

which is exactly AV@ $R_{\alpha}(\mathbf{X})$ when regarding $\mathbf{X}$ as "gain."

\section{Conflicts of Interest}

The authors declare that there are no conflicts of interest regarding the publication of this paper.

\section{References}

[1] P. Artzner, F. Delbaen, J.-M. Eber, and D. Heath, "Coherent measures of risk," Mathematical Finance, vol. 9, no. 3, pp. 203228, 1999.

[2] J. Haezendonck and M. Goovaerts, "A new premium calculation principle based on Orlicz norms," Insurance: Mathematics and Economics, vol. 1, no. 1, pp. 41-53, 1982.

[3] M. J. Goovaerts, R. Kaas, J. Dhaene, and Q. Tang, "Some new classes of consistent risk measures," Insurance: Mathematics and Economics, vol. 34, no. 3, pp. 505-516, 2004.

[4] F. Bellini and E. Rossaza Gianin, "On Heazendonck risk meausres," Journal of Banking \& Finance, vol. 32, pp. 986-994, 2008.

[5] F. Bellini and E. Rosazza Gianin, "Haezendonck-Goovaerts risk measures and Orlicz quantiles," Insurance: Mathematics and Economics, vol. 51, no. 1, pp. 107-114, 2012.

[6] A. S. Cherny, "Weighted V@R and its properties," Finance and Stochastics, vol. 10, no. 3, pp. 367-393, 2006.

[7] L. Chen, L. Lin, Y. Lu, and G. Parker, "Analysis of survivorship life insurance portfolios with stochastic rates of return," Insurance: Mathematics and Economics, vol. 75, pp. 16-31, 2017.

[8] O. Deprez and H. U. Gerber, "On convex principles of premium calculation," Insurance: Mathematics and Economics, vol. 4, no. 3, pp. 179-189, 1985.

[9] Z. Liang and K. C. Yuen, "Optimal dynamic reinsurance with dependent risks: variance premium principle," Scandinavian Actuarial Journal, no. 1, pp. 18-36, 2016.

[10] H. Meng, M. Zhou, and T. K. Siu, "Optimal dividendreinsurance with two types of premium principles," Probability in the Engineering and Informational Sciences, vol. 30, no. 2, pp. 224-243, 2016.

[11] A. Pichler, "Premiums and reserves, adjusted by distortions," Scandinavian Actuarial Journal, no. 4, pp. 332-351, 2015.

[12] C. Burgert and L. Rüschendorf, "Consistent risk measures for portfolio vectors," Insurance: Mathematics and Economics, vol. 38, no. 2, pp. 289-297, 2006.
[13] L. Rüschendorf, Mathematical Risk Analysis, Springer, 2013.

[14] E. Jouini, M. Meddeb, and N. Touzi, "Vector-valued coherent risk measures," Finance and Stochastics, vol. 8, no. 4, pp. 531552, 2004.

[15] A. H. Hamel, B. Rudloff, and M. Yankova, "Set-valued average value at risk and its computation," Mathematics and Financial Economics, vol. 7, no. 2, pp. 229-246, 2013.

[16] G. Ararat, A. H. Hamel, and B. Rudloff, "Set-valued shortfall and divergence risk measures," International Journal of Theoretical and Applied Finance, vol. 20, no. 5, 48 pages, 2017.

[17] Z. Feinstein and B. Rudloff, "Multi-portfolio time consistency for set-valued convex and coherent risk measures," Finance and Stochastics, vol. 19, no. 1, pp. 67-107, 2015.

[18] H. Föllmer and A. Schied, "Convex measures of risk and trading constraints," Finance and Stochastics, vol. 6, no. 4, pp. 429-447, 2002.

[19] A. H. Hamel, "A duality theory for set-valued functions. I. Fenchel conjugation theory," Set-Valued and Variational Analysis, vol. 17, no. 2, pp. 153-182, 2009.

[20] A. H. Hamel and F. Heyde, "Duality for set-valued measures of risk," SIAM Journal on Financial Mathematics, vol. 1, no. 1, pp. 66-95, 2010. 


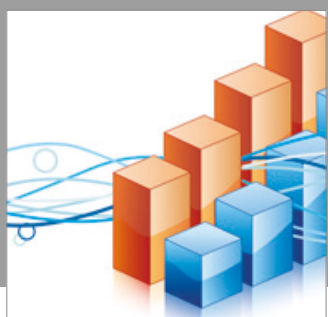

Advances in

Operations Research

vatersals

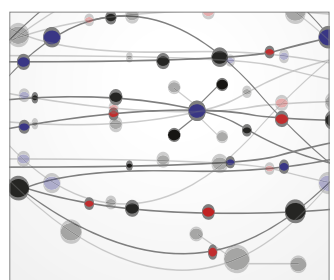

\section{The Scientific} World Journal
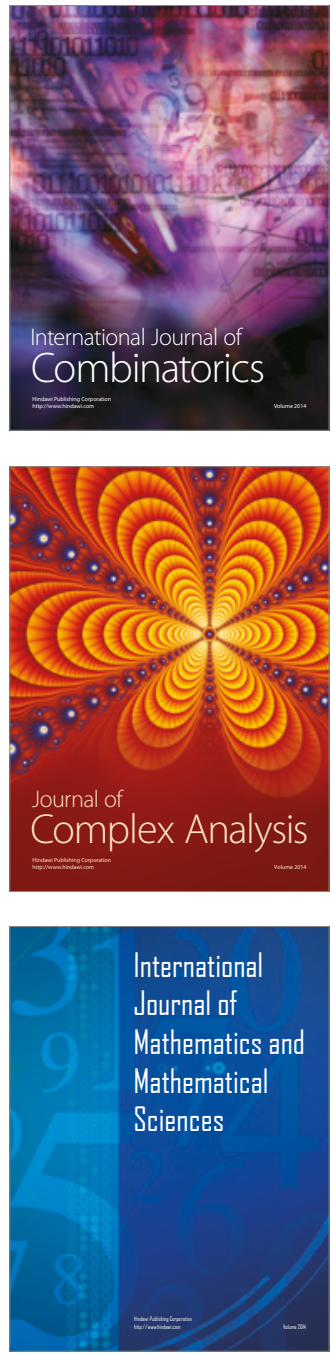
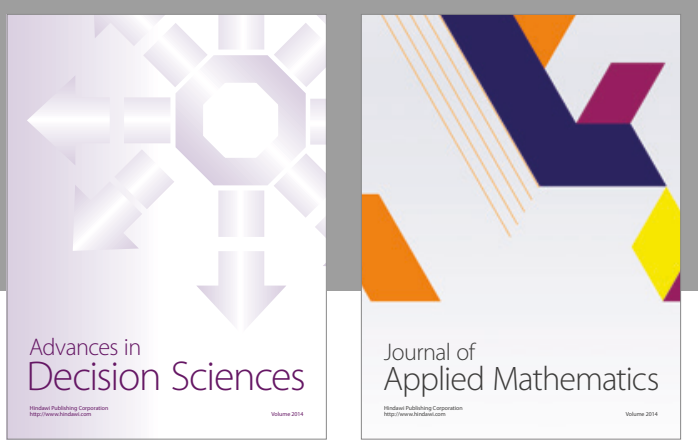

Algebra

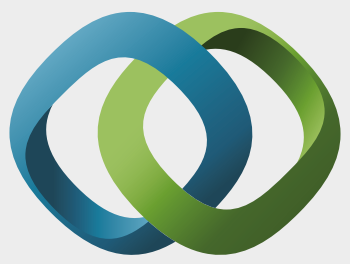

\section{Hindawi}

Submit your manuscripts at

https://www.hindawi.com
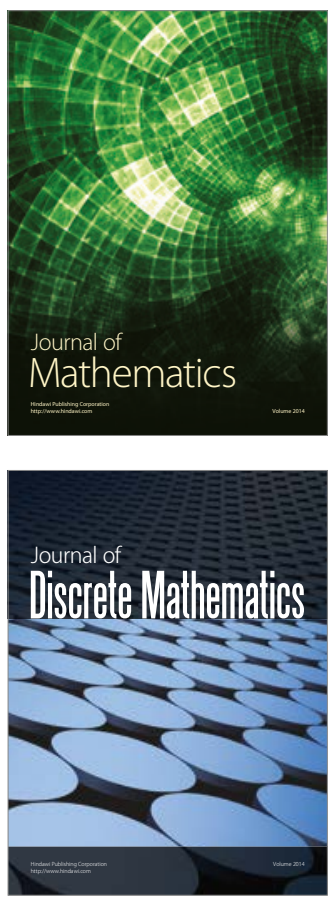

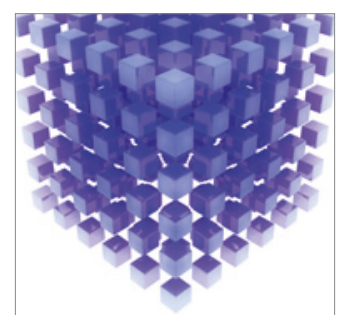

Mathematical Problems in Engineering
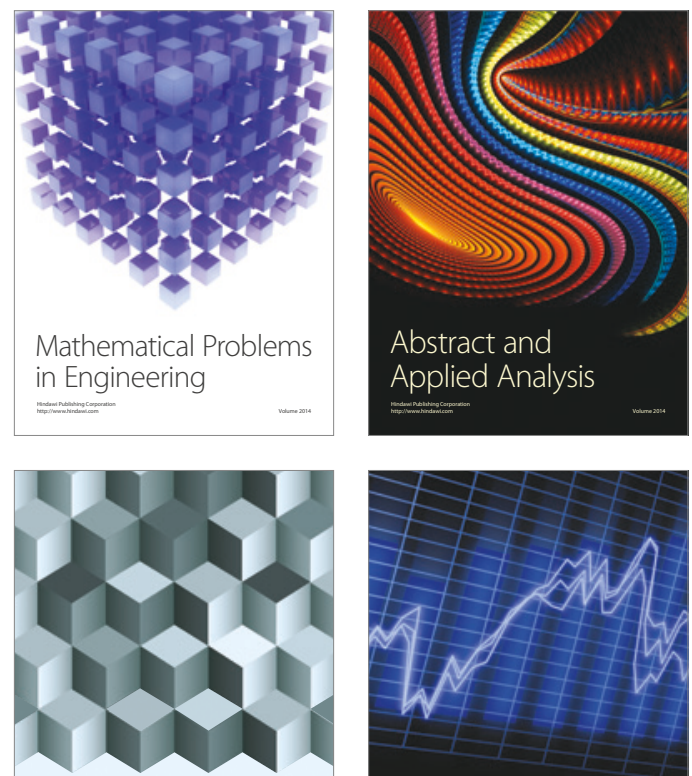

Journal of

Function Spaces

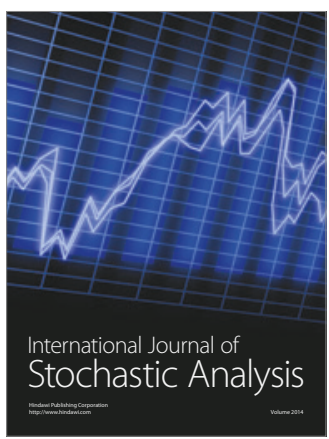

Probability and Statistics
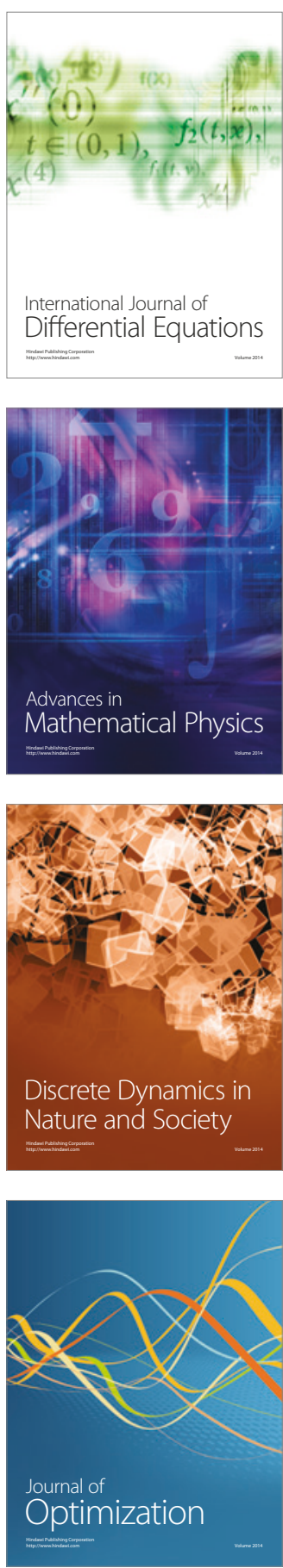